\title{
Clinical and Neuropsychological Correlates of White Matter Abnormalities in Recent Onset Schizophrenia
}

\author{
Philip R Szeszko*,1,2,3, Delbert G Robinson ${ }^{1,2,3}$, Manzar Ashtari', Joshua Vogel', Julia Betensky', \\ Serge Sevy ${ }^{1,2}$, Babak A Ardekani ${ }^{4}$, Todd Lencz ${ }^{1,2,3}$, Anil K Malhotra ${ }^{1,2,3}$, Joanne McCormack', \\ Rachel Miller', Kelvin O Lim ${ }^{5}$, Handan Gunduz-Bruce ${ }^{6}$, John M Kane ${ }^{1,2,3}$ and Robert M Bilder \\ 'Department of Psychiatry Research, The Zucker Hillside Hospital, North Shore-Long Island Jewish Health System, Glen Oaks, NY, USA; \\ ${ }^{2}$ Department of Psychiatry, Albert Einstein College of Medicine, Bronx, NY, USA; ${ }^{3}$ Feinstein Institute for Medical Research, North Shore-Long \\ Island Jewish Health System, Manhasset, NY, USA; ${ }^{4}$ Nathan S Kline Institute for Psychiatric Research, Center for Advanced Brain Imaging, \\ Orangeburg, NY, USA; ${ }^{5}$ Department of Psychiatry, University of Minnesota, Minneapolis, MN, USA; ${ }^{6}$ Department of Psychiatry, Yale University \\ School of Medicine, New Haven, CT, USA; ${ }^{7}$ UCLA Neuropsychiatric Institute and Geffen School of Medicine, Los Angeles, CA, USA
}

\begin{abstract}
The objective of this study was to investigate the clinical and neuropsychological correlates of white matter abnormalities in patients with schizophrenia studied early in the course of illness. A total of 33 (2I male/ 12 female) patients with recent onset schizophrenia and 30 ( 8 male//2 female) healthy volunteers completed structural and diffusion tensor imaging exams. Patients also received clinical and neuropsychological assessments. Fractional anisotropy (FA) maps were compared between groups in the white matter using a voxelwise analysis following intersubject registration to Talairach space and correlated with functional indices. Compared to healthy volunteers, patients demonstrated significantly $(p<0.00$ I, cluster size $\geqslant 100$ ) lower FA within temporal lobe white matter regions corresponding approximately to the right and left uncinate fasciculus, left inferior fronto-occipital fasciculus, and left superior longitudinal fasciculus. There were no areas of significantly higher FA in patients compared to healthy volunteers. Lower FA in the bilateral uncinate fasciculus correlated significantly with greater severity of negative symptoms (alogia and affective flattening), and worse verbal learning/memory functioning. In addition, higher FA in the inferior fronto-occipital fasciculus correlated significantly with greater severity of delusions and hallucinations. White matter abnormalities are evident in patients with schizophrenia early in the course of illness, appearing most robust in left temporal regions. These abnormalities have clinical and neuropsychological correlates, which may be useful in further characterizing structure-function relations in schizophrenia and constraining neurobiological models of the disorder.

Neuropsychopharmacology (2008) 33, 976-984; doi:I 0.1038/sj.npp. I 30I480; published online 20 June 2007
\end{abstract}

Keywords: schizophrenia; white matter; diffusion tensor imaging; symptoms; neuropsychology; temporal lobes

\section{INTRODUCTION}

There is increasing evidence that schizophrenia involves structural alterations in the brain white matter and that a defect in this connectivity contributes to the pathophysiology of the disorder (Davis et al, 2003). Post-mortem studies have identified abnormalities in the myelin sheath (Uranova et al, 2001) and oligodendroglia (Hof et al, 2003) in patients compared to healthy volunteers. Decreased expression of several myelination-related genes in patients further supports the hypothesis of white matter abnormalities in schizophrenia (Hakak et al, 2001). Moreover, a meta-

*Correspondence: Dr PR Szeszko, Department of Psychiatry Research, The Zucker Hillside Hospital, 75-59 263rd Street, Glen Oaks, NY | |004, USA, Tel: + | 718470 8489, Fax: + | 718343 |659,

E-mail: szeszko@lij.edu

Received 22 January 2007; revised II May 2007; accepted 17 May 2007 analysis of magnetic resonance imaging (MRI) studies also implicated white matter abnormalities in patients at the gross anatomic level, indicating that they are more pronounced in frontal and temporal lobe regions (Wright et al, 2000).

Diffusion tensor imaging (DTI) is an MRI technique that may have implications for understanding the anatomical organization of axons and myelin sheath and thus represents a potentially powerful tool for in vivo mapping of anatomical connectivity in humans. If the pathophysiology of schizophrenia reflects a disturbance in the white matter, then such abnormalities might be observed using DTI. Early studies showed widespread anisotropic alterations in the integrity of prefrontal white matter (Buchsbaum et al, 1998) and whole-brain white matter (Lim et al, 1999). Subsequent studies reported lower anisotropic diffusion within specific regions including the corpus callosum (Ardekani et al, 2003; Kubicki et al, 2005), internal capsule (Kubicki et al, 2005; Szeszko et al, 2005a; Buchsbaum et al, 
2006; Mitelman et al, 2007), anterior cingulate (Kumra et al, 2005; Wang et al, 2004; Kubicki et al, 2003), arcuate fasciculus (Kubicki et al, 2005; Burns et al, 2003), and inferior longitudinal fasciculus (Hubl et al, 2004). Additional studies reported a lack of normal asymmetry in white matter regions such as the internal capsule (Park et al, 2004), uncinate fasciculus (Park et al, 2004; Kubicki et al, 2002), and superior longitudinal fasciculus (Park et al, 2004) in patients compared to healthy volunteers and that internal capsule abnormalities are associated with worse outcome among patients (Brickman et al, 2006).

The majority of prior DTI studies in schizophrenia may be limited, however, by the use of chronic patients treated for long periods of time with antipsychotic medications. Examination of these abnormalities closer to illness onset in schizophrenia may be an important consideration, especially given evidence for a progressive reduction in white matter volume over the course of illness (Ho et al, 2003). Moreover, although white matter microstructural abnormalities have been identified in schizophrenia their functional significance remains largely unknown. In this study we report a voxelwise analysis of DTI data in patients with recent onset schizophrenia compared to healthy volunteers and their clinical and neuropsychological correlates. We hypothesized that patients would demonstrate lower fractional anisotropy (FA) compared to healthy volunteers in the frontal and temporal lobe white matter. Moreover, consistent with prior work linking neuropsychological measures to structural deficits in frontotemporal regions (Antonova et al, 2004; Lim et al, 2006; Nestor et al, 2004), we predicted that lower FA in the frontal and temporal lobes would be associated with worse executive and memory functioning, respectively.

\section{METHODS}

\section{Subjects}

The 33 patients included in this study were recruited from admissions to the inpatient service at The Zucker Hillside Hospital in Glen Oaks, NY. All but two patients had participated in a clinical treatment trial comparing the efficacy of risperidone $v s$ olanzapine. Further details regarding the ascertainment of patients and the clinical trial are available in Robinson et al (2006). In the present study we defined recent onset as having a duration of illness from the index episode or entry into the clinical trial to the MRI exam of 4.25 years or less (median $=79$ weeks, mode $=0$, range $=0-4.25$ years). The mean number of weeks from hospitalization and the initiation of antipsychotic treatment to the DTI exam was $77(\mathrm{SD}=78)$. Patients had minimal antipsychotic drug exposure before the initiation of treatment in the clinical trial: 27 had no prior treatment, 1 had 2 weeks of treatment with risperidone, 2 had 2 days of treatment ( 1 with risperidone and 1 with haldol and olanzapine), 1 had 3 days of treatment with haldol, 1 had 4 days of treatment with haldol and risperidone, and 1 had 6 days of treatment (antipsychotic unknown). Diagnoses were based on the SCID for Axis I DSM-IV disorders supplemented by information from family members and clinicians. All patients met DSM-IV criteria for schizophrenia $(n=25)$, schizoaffective disorder $(n=4)$, or schizophreniform disorder $(n=4)$. All patients received a physical exam and laboratory screening to rule out medical causes of their psychotic episode. Eleven patients had a comorbid diagnosis of substance abuse and seven patients were antipsychotic drug naive at the time of their scan. Mean age at first psychotic symptoms was 20.5 $(\mathrm{SD}=4.2)$. In addition, 33 healthy comparison subjects were recruited from local newspaper advertisements and through word of mouth in the community. Exclusion criteria for healthy subjects included the denial of any Axis I psychiatric disorder (lifetime) and having a first-degree relative with psychosis as determined by clinical interview and the SCID-NP. Exclusion criteria for all study participants included metal implants, any serious neurologic or endocrine disorder, or meeting DSM-IV criteria for mental retardation. This study was approved by the North ShoreLong Island Jewish Medical Center Institutional Review Board and written informed consent was obtained from all study participants.

\section{Handedness}

Classification of handedness was based on a modified version of the Edinburgh Inventory consisting of 20 items. Total number of right and left hand items was scored and the laterality quotient was computed according to the following formula: (Total R-Total L)/(Total R+Total L). This yielded a total laterality quotient for each subject that ranged from +1.00 (totally dextral) to -1.00 (totally nondextral). Subjects with a laterality quotient greater than 0.70 were classified as dextral, and the rest as nondextral.

\section{Clinical Assessments}

We computed two symptom cluster scores based on items from the Scale for the Assessment of Negative Symptoms (SANS) and Schedule for Affective Disorders and Schizophrenia - change version with psychosis and disorganization items (SADS-C + PD). The positive symptom cluster score was computed as the average of the severity of delusions and severity of hallucinations items from the SADS-C+PD. The negative symptom cluster score was computed as the average of the global ratings of affective flattening, alogia, avolition apathy, and asociality from the SANS. Intraclass correlation coefficients among three psychopathology raters for the SADS-C + PD severity of delusions item was 0.79 and for the severity of hallucinations item was 0.90. Intraclass correlation coefficients for the global items of the SANS ranged from 0.66 to 0.82 .

\section{Neuropsychological Assessments}

To minimize Type-I error we computed five neuropsychological domains a priori based on what the individual tests purport to measure, a strategy employed by our group previously in first-episode schizophrenia (Bilder et al, 2000), childhood-onset schizophrenia (Rhinewine et al, 2005), and prodromal schizophrenia (Lencz et al, 2006). Each domain was computed as the average of $z$-scores of individual tests comprising the respective domain. Scores were computed so that higher values were indicative of better performance. Domains (with coefficient $\alpha$ in 
parentheses) included: (1) verbal learning/memory (0.86) comprising animal naming and Wechsler Memory Scale-Revised Logical Memory I and II; (2) visual memory (0.85) comprising Wechsler Memory Scale-Revised Visual Reproduction I and II; (3) motor (0.77) comprising right- and left-handed finger tapping and grooved pegboard performance; (4) attention (0.78) comprising trail making test part A total time, cancellation test total time, and Wechsler Adult Intelligence Scale-digit span score; and (5) executive (0.86) comprising Wisconsin Card Sorting Test (WCST) categories, WCST perseverative errors, and trail making test part B total time.

\section{Magnetic Resonance Imaging Procedures}

MRI examinations were conducted at the Long Island Jewish Medical Center on a General Electric $1.5 \mathrm{~T}$ whole body superconducting imaging system. All scans were reviewed by a neuroradiologist for gross pathology, which would preclude participation in this study. Scans were also reviewed by a member of the research team, and any scan with significant artefacts was repeated. We minimized movement by stabilizing the head with cushions and tape before scanning.

A total of 26 DTI volumes were obtained from each subject that included 25 volumes with diffusion gradients applied along 25 nonparallel directions with $b=1000 \mathrm{~s} / \mathrm{mm}^{2}$ and $\mathrm{NEX}=2$, and one volume without diffusion weighting $(b=0 ; \mathrm{NEX}=2)$. Each volume consisted of 23 contiguous $5 \mathrm{~mm}$ axial slices acquired parallel to the anterior-posterior commissural line using a ramp sampled, spin-echo, single-shot echo-planar imaging (EPI) method (TR $=10 \mathrm{~s}$, $\mathrm{TE}=83.3 \mathrm{~ms}, 128 \times 128$ matrix, field of view $(\mathrm{FOV})=22 \mathrm{~cm})$. We did not find any evidence for systematic ghosting on the diffusion tensor images as determined from visual inspection of images before analysis.

To provide a high-resolution anatomical reference, 124 contiguous coronal images (slice thickness $=1.5 \mathrm{~mm}$ ) were acquired through the whole head using a 3D Fast SPGR sequence with IR Prep $(\mathrm{TR}=10.1 \mathrm{~ms}, \mathrm{TE}=4.3 \mathrm{~ms}$, $\mathrm{TI}=600 \mathrm{~ms}, \quad \mathrm{FOV}=22 \mathrm{~cm}, 256 \times 256$ matrix) producing nominal in-plane resolution of $0.86 \times 0.86 \mathrm{~mm}$. In addition, an oblique axial fast spin echo scan $(\mathrm{TR}=4 \mathrm{~s}, \mathrm{TE}=$ $20 / 100 \mathrm{~ms}, \mathrm{FOV}=22 \mathrm{~cm}, 256 \times 256$ matrix) was acquired at the same slice positions as the diffusion tensor images and provided contiguous $5 \mathrm{~mm}$ thick proton density (PD) and T2-weighted (T2) images. The fast spin echo PD and T2 volumes were used for rigid-body registration and distortion correction of diffusion images and for image segmentation.

\section{Image Processing}

Image processing was conducted using three image registration steps based on previously published methods (Szeszko et al, 2005a,b). Briefly, nonbrain regions were removed from the SPGR images using the brain extraction tool of the FSL software package; these results were improved manually using the MEDx software package (v. 3.4; MEDx, 2000). Total intracranial volumes were computed for all individuals; the median volume was transformed into Talairach space using the AFNI software package and used as the target (template) image. SPGR volumes from the other study participants were matched to the target volume using an in-house nonlinear registration software program (Ardekani et al, 2005). For all individuals the cropped SPGR volume was registered to their fast spin echo T2/PD volumes (Ardekani et al, 1995) yielding a resliced SPGR volume with the same orientation and voxel size as the T2/PD volumes. These resliced SPGR volumes were used as masks to delete nonbrain regions from the T2/PD volumes of the respective subject. The resulting image sets (ie cropped T2/PD and cropped and resliced SPGR) were used as three channels in the FSL-FAST software package to create a white matter mask for each subject following segmentation of the brain into white matter, gray matter, and cerebrospinal fluid (Figure 1). A mask is defined as a binary image in which the voxels have a value of 1 if they belong to the tissue of interest and 0 otherwise. Individuals' white matter masks were transformed to Talairach space and averaged and thresholded at $40 \%$ to obtain a white matter mask for the entire group. Voxelwise statistical analyses were confined to the voxels comprising this white matter mask. To correct for spatial distortion of the DTI EPI data, we registered the $b=0$ DTI volume to the cropped T2 volume using an in-house nonlinear registration program (Ardekani et al, 2005; Figure 2).

For all individuals an FA map was computed from the DTI volumes following derivation of the eigenvalues of the diffusion tensor matrix for each voxel using methods described previously by Basser (1995). The diffusion tensor and FA maps were computed in the original native

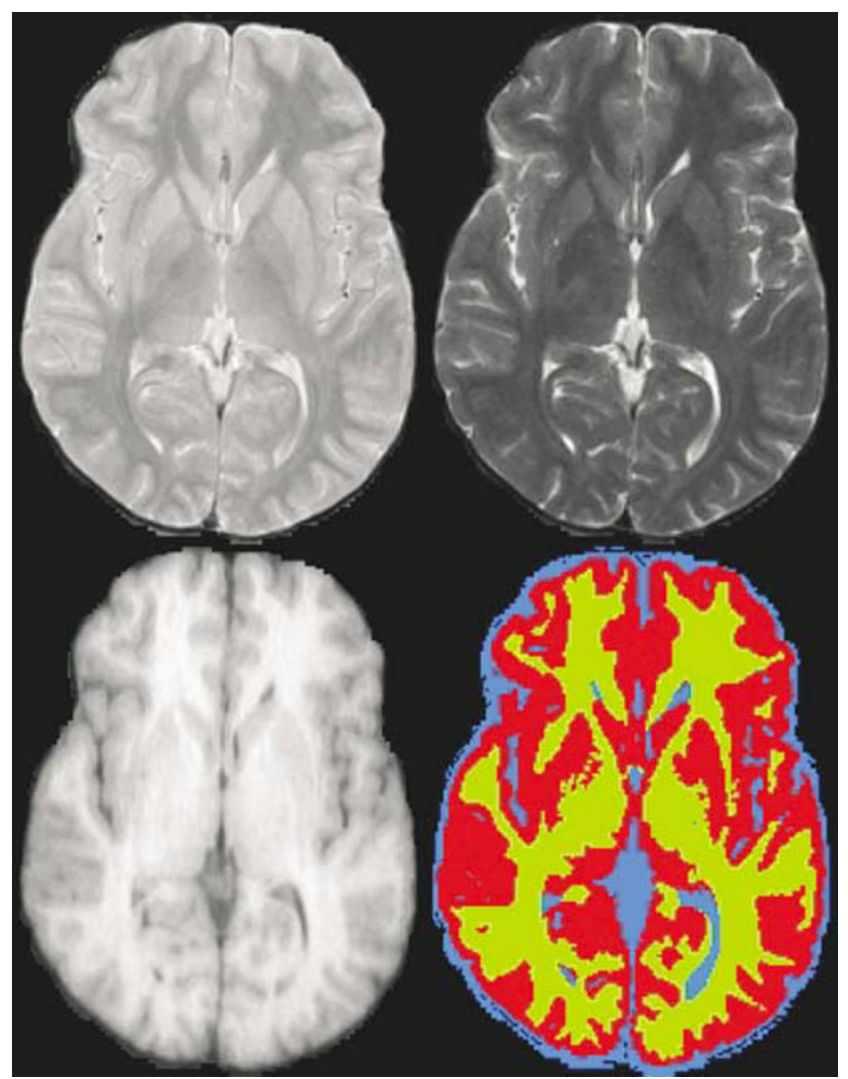

Figure I Illustration of the three-channel segmentation. 


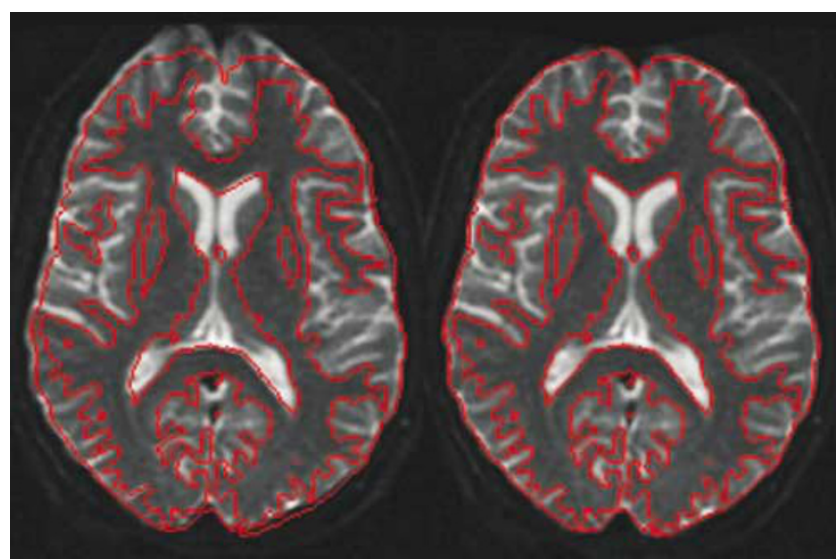

Figure 2 White matter boundaries superimposed on the original $b=0$ DTI slice (left) and on the distortion-corrected DTI slice (right). Note the distortion in the anterior and posterior regions that has been corrected.

coordinates of the acquired images before any registration operations were applied to the scalar values. The FA map of each subject was transformed into Talairach space by combining the three transformations described above: (1) intersubject nonlinear deformation of the SPGR volume to the target volume; (2) intrasubject linear rigid-body transformation of the SPGR volume to the T2/PD volume; and (3) nonlinear intrasubject registration of the DTI to the T2 volume for distortion correction. The resulting transformation was then applied to the original FA map by a single interpolation operation. We, therefore, obtained FA maps of matrix size $161 \times 191 \times 151$ and voxel size $1 \times 1 \times 1 \mathrm{~mm}^{3}$ in common Talairach space. Both the registered FA and white matter images were smoothed with a 3D isotropic Gaussian kernel with $\sigma=6 \mathrm{~mm}$. Identification of specific white matter tracts was made using the MRI Atlas of Human White Matter by Mori et al (2005).

\section{Statistical Analyses}

Group differences in demographic variables were examined using independent groups $t$-tests. $\chi^{2}$-tests were used to examine differences in joint classifications of discrete variables. Two-sample $t$-tests were performed at each voxel on the FA values within the brain white matter between patients and controls. Voxels that had a $t$-statistic greater than $3.22(p<0.001$; two-tailed $)$ and were part of a spatially contiguous cluster size of 100 voxels or greater were considered to have significantly different FA in patients compared to healthy volunteers. This $\alpha$ level and cluster size was chosen to maintain a balance between Type-I and TypeII error rate given the potential for increased Type-I error due to multiple comparisons, but recognizing that tests at adjacent voxels are strongly interdependent (Forman et al, 1995) and for consistency with our six-direction DTI pilot study in first-episode schizophrenia (Szeszko et al, 2005a). Partial correlation analysis was used to examine the relationship between FA and the neuropsychological measures while controlling for education level. Given that patients were at various stages of treatment, we used antipsychotic treatment duration as a covariate in partial correlation analyses investigating FA in relation to positive and negative symptoms. All scatterplots were inspected for possible outliers that could unduly influence results. We used the method described by Meng et al (1992) to compare the magnitude of correlation coefficients and test for specificity of structure-function relations. As a further test of selectivity among patients, we examined the relationship between the white matter regions that differed significantly between groups (independent variables) as predictors of each domain of neuropsychological functioning using multiple regression. Complete clinical and neuropsychological data were unavailable for two patients. In addition, one patient was missing data for WCST perseverative errors and another patient was missing data for left-handed grooved pegboard performance. Additional analyses investigated the potential effects of sex, education, duration of psychosis, antipsychotic drug exposure, and substance abuse history on the observed findings.

\section{RESULTS}

Patients and comparison subjects did not differ significantly in distributions of age, sex, laterality quotient, parental social class, or race (see Table 1). Moreover, the subgroup of antipsychotic drug-naive patients did not differ significantly in neuropsychological functioning from patients being treated with antipsychotics in any of the neuropsychological domains, but as expected, had significantly greater positive and negative symptoms.

Clusters of at least 100 contiguous voxels $(p<0.001)$ superimposed on the average normalized SPGR for all subjects are illustrated in Figure 3. Significantly decreased FA in patients compared to healthy volunteers was observed in four regions in the temporal lobe white matter (see Figure 3 and Table 2). Repeated measures analysis of covariance using education as a covariate indicated that patients had significantly lower FA across these 4 regions $(F(1,60)=13.15, p=0.001)$. Neither the main effect of sex nor the group-by-sex interaction was statistically significant. There were no areas of significantly higher FA in patients compared to healthy volunteers at this threshold and cluster size.

Lower FA in the uncinate fasciculus bilaterally correlated significantly with greater severity of negative symptoms ( $r=-0.36, \mathrm{df}=31, p=0.048)$, which remained significant while controlling for antipsychotic treatment duration $(r=-0.36, \mathrm{df}=28, p=0.047$; Figure 4). Post hoc tests revealed significant correlations of uncinate fasciculus FA with the global rating of alogia $(r=-0.43, \mathrm{df}=28$, $p=0.016)$ and the global rating of affective flattening $(r=-0.46, \mathrm{df}=31, p=0.009)$. In addition, higher FA in the inferior fronto-occipital fasciculus correlated significantly with greater severity of positive symptoms $(r=0.60$, $\mathrm{df}=31, p<0.001)$, which remained significant while controlling for duration of antipsychotic treatment $(r=0.60$, $\mathrm{df}=28, \quad p<0.001$; Figure 5). Post hoc tests revealed significant effects for the global rating of hallucinations $(r=0.64, \mathrm{df}=31, p<0.001)$ and the global rating of delusions $(r=0.52, \mathrm{df}=31, p=0.003)$. Post hoc investigation of individual hallucination and delusion items on the SADS-C + PD revealed that higher FA in this region was significantly correlated with greater severity of auditory hallucinations $(r=0.57, \mathrm{df}=31, p=0.001)$, tactile 
Table I Sample Characteristics

\begin{tabular}{|c|c|c|c|c|c|}
\hline Value & Patients with schizophrenia $(N=33)$ & Healthy volunteers $(N=30)$ & df & Test statistics & $p$-value \\
\hline Age & $25.1(4.1)$ & $25.9(4.6)$ & 61 & $t=0.69$ & NS \\
\hline Sex (male/female) & $21 / 12$ & $18 / 12$ & । & $\chi^{2}=0.09$ & NS \\
\hline Parental social class ${ }^{\mathrm{a}, \mathrm{b}}$ & $2.8(0.76)$ & $2.6(0.63)$ & 60 & $\chi^{2}=2.0$ & NS \\
\hline Laterality quotient & $0.76(0.54)$ & $0.87(0.36)$ & 60 & $t=0.94$ & NS \\
\hline Race $^{c}$ & $(4,22,0,5,2)$ & $(8,12,4,4,2)$ & 2 & $\chi^{2}=2.0$ & NS \\
\hline
\end{tabular}

Abbreviation; NS, not significant.

Data are presented as mean $\pm S D$ in parentheses, unless otherwise indicated. There were data were missing for the following variables: parental social class (one patient), years of education (three patients, one healthy volunteer), and laterality quotient (one healthy volunteer).

aHollingshead Redlich Scale.

bParental social class was rated on a scale from I (highest) to 5 (lowest). Due to the low number of expected frequencies in groups I and 5 , groups I and 2 were combined, and groups 4 and 5 were combined for analysis.

${ }^{c}$ Race coded as Caucasian, African-American, Hispanic, Asian, and other. Because more than 20\% of the categories for race had expected frequencies of less than 5 we combined the latter three groups (ie Hispanic, Asian, and other) into a single group for analysis.

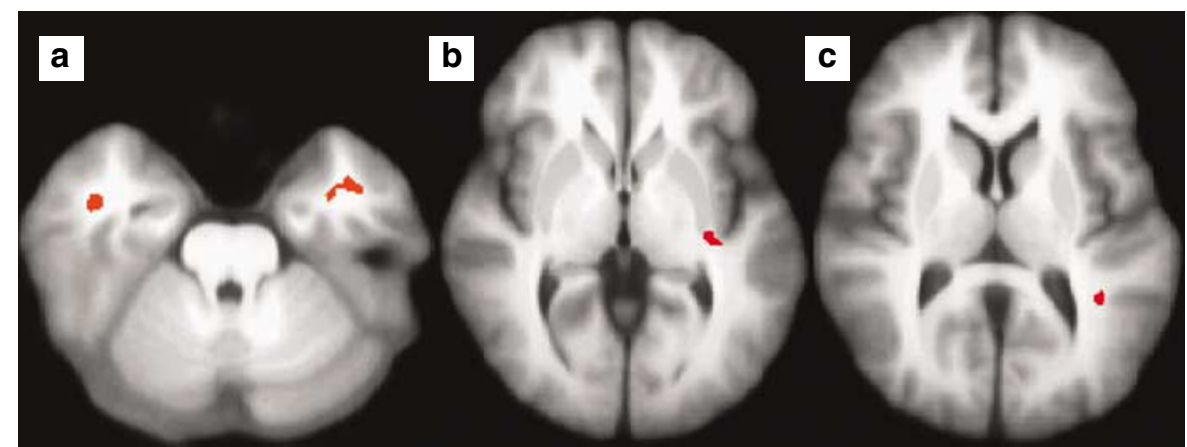

Figure 3 White matter regions in which FA was significantly $(p<0.00 \mathrm{I} ; \mathrm{I} 00$ voxels) lower in patients compared to healthy volunteers in the right and left uncinate fasciculus (a), inferior fronto-occipital fasciculus (b), and superior longitudinal fasciculus (c). A description of these regions can be found in the text and Table 2 .

Table 2 Mean Fractional Anisotropy Values for Patients and Healthy Volunteers

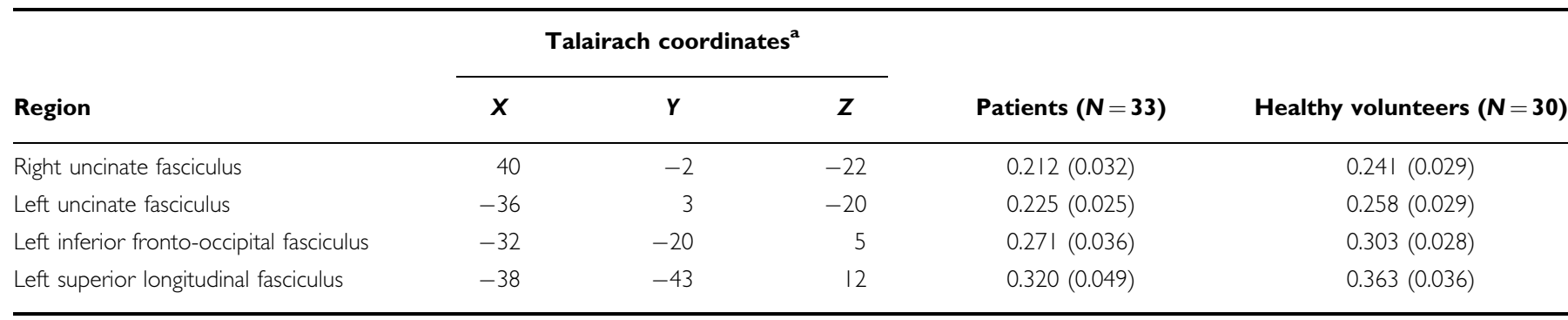

${ }^{\text {a }}$ Talairach coordinates represent the centroid of each region.

hallucinations $(r=0.52, \mathrm{df}=31, p=0.003)$, visual hallucinations $(r=0.37, \mathrm{df}=31, p=0.04)$, delusions of being controlled $(r=0.44, \mathrm{df}=31, p=0.014)$, delusions of mind reading $(r=0.46, \mathrm{df}=31, p=0.01)$, persecutory delusions $(r=0.53, \mathrm{df}=31, p=0.002)$, and delusions of reference $(r=0.42, \mathrm{df}=31, p=0.02)$. Neither positive nor negative symptoms correlated significantly with FA in the superior longitudinal fasciculus.

Correlations of NP domains and white matter regions that differed between groups are illustrated in Table 3. Lower FA in the uncinate fasciculus bilaterally correlated significantly with worse verbal learning/memory $(r=0.46, \mathrm{df}=31$, $p=0.009)$, which remained significant while controlling for education $(r=0.41, \mathrm{df}=28, p=0.025$; Figure 6$)$. This finding was more robust for the right $(r=0.34, \mathrm{df}=28$, $p=0.06)$ compared to the left $(r=0.25, \mathrm{df}=28, p=0.18)$ hemisphere, although the magnitude of these correlations did not differ significantly from each other. Testing the specificity of structure-function relations revealed that FA in the uncinate fasciculus correlated significantly more strongly with verbal learning/memory functioning compared to performance on the visual memory $(z($ diff $)=3.26$, 


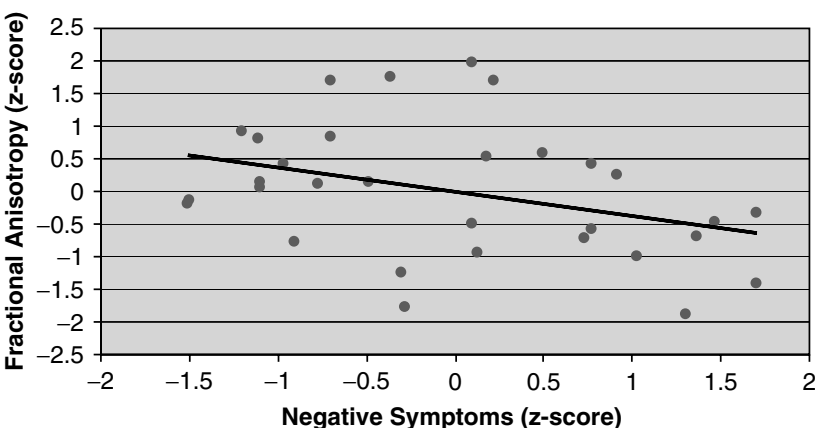

Figure 4 Scatterplot of FA in the bilateral uncinate fasciculus and negative symptoms in patients controlling for antipsychotic treatment duration.

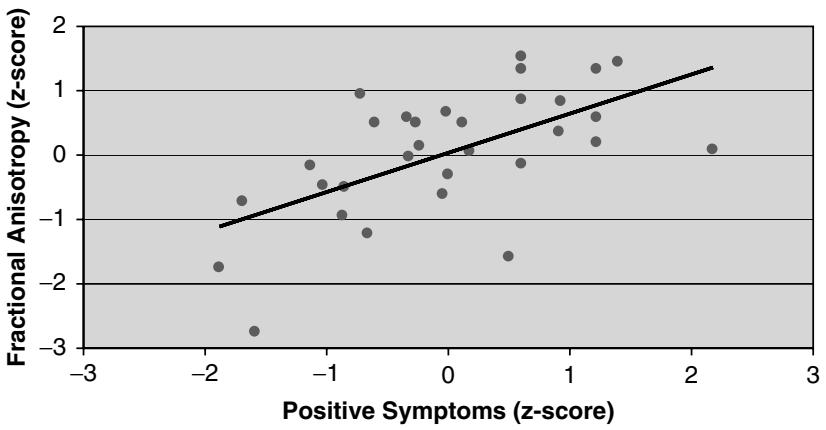

Figure 5 Scatterplot of FA in the left inferior fronto-occipital fasciculus and positive symptoms in patients controlling for antipsychotic treatment duration.

Table 3 Correlations of Neuropsychological Domains with Areas of Abnormal Fractional Anisotropy in Patients

\begin{tabular}{lccc}
\hline & $\begin{array}{c}\text { Bilateral } \\
\text { uncinate } \\
\text { fasciculus }\end{array}$ & $\begin{array}{c}\text { Left inferior } \\
\text { fronto-occipital } \\
\text { fasciculus }\end{array}$ & $\begin{array}{c}\text { Left superior } \\
\text { longitudinal } \\
\text { fasciculus }\end{array}$ \\
\hline Attention & -0.03 & -0.13 & 0.15 \\
Executive & -0.15 & -0.18 & 0.35 \\
Motor & -0.07 & -0.02 & 0.12 \\
Verbal memory & $0.46 *$ & -0.05 & 0.25 \\
Visual memory & -0.17 & -0.08 & 0.26 \\
\hline
\end{tabular}

* $p<0.01$.

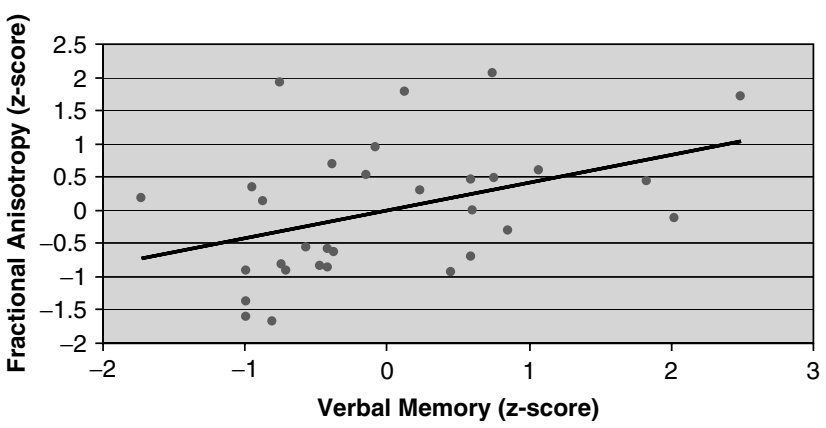

Figure 6 Scatterplot of FA in the bilateral uncinate fasciculus and verbal memory functioning in patients controlling for education. $\mathrm{df}=31, p<0.001)$, motor $(z(\operatorname{diff})=2.50, \mathrm{df}=30, p<0.005)$, executive $(z$ (diff $)=3.03, \mathrm{df}=30, p<0.001)$, and attention $(z(\mathrm{diff})=2.92, \mathrm{df}=31, p<0.001)$ domains. Post hoc investigation of the individual tests revealed that lower FA in the uncinate fasciculus correlated significantly with worse Logical Memory I $(r=0.37, \mathrm{df}=31, p=0.041)$, Logical Memory II $(r=0.37, \mathrm{df}=31, p=0.042)$, and animal naming $(r=0.49, \mathrm{df}=31, p=0.005)$ performance. None of the neuropsychological domains correlated significantly with FA in the left inferior fronto-occipital fasciculus or superior longitudinal fasciculus. Multiple regression revealed that the overall model predicting verbal learning/memory using the white matter regions that differed significantly between groups as independent variables was significant $(\mathrm{F}=3.29$, $\mathrm{df}=3,27, \quad p=0.036)$; however, the bilateral uncinate fasciculus region was the only one that significantly predicted verbal learning/memory performance $(t=2.71$, $\beta=0.45, p=0.012)$. None of the multiple regressions predicting any other neuropsychological domain using the white matter regions that differed significantly between groups as independent variables was significant.

Additional analyses investigated the potential effects of illness duration, prior antipsychotic drug exposure, and substance abuse history on the observed findings. There were no significant correlations between age at first psychotic symptoms and FA in any of the four regions that differed significantly between patients and healthy volunteers. Additional analyses revealed that both antipsychotic drug-naive patients $(\mathrm{F}=10.05, \mathrm{df}=1,35, p=0.003)$ and patients without any history of substance abuse $(\mathrm{F}=59.71$, $\mathrm{df}=1,50, p<0.001)$ had significantly lower FA across the four regions compared to healthy volunteers; the group-byregion interaction was not statistically significant in either analysis. We found no significant effect of group for antipsychotic treatment duration when we used a median split to categorize patients into short $v s$ long antipsychotic drug exposure. Moreover, antipsychotic drug-naive patients did not differ significantly from patients exposed to antipsychotic medications in FA for the four regions identified as abnormal in the overall sample. Exploratory correlations, however, revealed that antipsychotic treatment duration was significantly correlated with $\mathrm{FA}$ in the superior longitudinal fasciculus $(r=-0.44, \mathrm{df}=33$, $p=0.011$ ), but not with FA in any of the other three regions.

\section{DISCUSSION}

Our findings suggest that patients with schizophrenia studied early in the course of illness exhibited lower anisotropic diffusion in temporal lobe white matter regions corresponding approximately to the uncinate fasciculus, inferior fronto-occipital fasciculus, and superior longitudinal fasciculus. Lower anisotropic diffusion in the brain white matter of patients could be associated with either micro- or macrostructural alterations involving the myelin sheath and/or directional coherence of fiber tracts. Several post-mortem studies reported structural abnormalities in the oligodendroglia (Hof et al, 2003), which could be associated with changes in tissue water mobility as assessed via DTI. Moreover, microarray studies reported downregulation of oligodendrocyte and myelination genes as well 
as alterations in transcription factors believed to regulate gene expression (Tkachev et al, 2003), which also purportedly play a role in white matter development. In that regard it may therefore be noteworthy that Bartzokis et al (2003) reported that patients with schizophrenia lacked the normal age-associated decreases in gray to white matter ratios in the frontal and temporal lobes implicating a defect in white matter volume expansion among patients.

In the present study white matter abnormalities were evident mainly in left temporal regions in patients compared to healthy volunteers consistent with hypotheses regarding the failure of left hemisphere lateralization in the pathophysiology of schizophrenia (Crow, 1999). Our findings thus converge with Kubicki et al (2002) and Burns et al (2003) who reported abnormal anisotropic diffusion in the left hemisphere white matter in patients with schizophrenia. There is considerable evidence for asymmetry in several temporal lobe regions (eg the planum temporale) in healthy humans reflecting their importance in language processing (Galaburda et al, 1978). Interestingly, language abnormalities have been reported to be some of the core deficits observed in schizophrenia (Szeszko et al, 1999; Ruchsow et $a l, 2003)$ and have been associated with abnormal temporal gray matter asymmetry in schizophrenia (Jacobsen et al, 1997). Our findings also converge with prior volumetric MRI studies reporting less left hemisphere superior temporal gray matter in both chronic (Kwon et al, 1999) and first-episode (Hirayasu et al, 2000) patients compared to healthy volunteers. It is also of interest that the centroid of the two posterior left temporal white matter regions identified as abnormal in the present study, which included the inferior fronto-occipital fasciculus and superior longitudinal fasciculus, was within 13 and $11.9 \mathrm{~mm}$, respectively from the centroid of the superior temporal white matter region identified as abnormal in our prior sixdirection DTI study (Szeszko et al, 2005a).

The finding that patients had abnormal white matter in the uncinate fasciculus is consistent with results from several prior DTI studies in schizophrenia that used older samples (Kubicki et al, 2002; Burns et al, 2003) and with hypotheses regarding abnormal frontotemporal connectivity playing a role in the pathophysiology of the disorder (Szeszko et al, 2002, 2003; Bilder et al, 1995). Investigation of the functional correlates of FA in the uncinate fasciculus revealed significant inverse correlations with negative symptoms, including alogia and affective flattening. Our results thus converge with prior studies reporting an association between temporal gray (Anderson et al, 2002) and white (Sigmundsson et al, 2001) matter volumetric deficits and negative symptoms in patients with schizophrenia. Moreover, lower FA in this region correlated significantly with worse memory functioning. This finding is consistent with Nestor et al (2004) who reported that lower FA in the uncinate fasciculus correlated significantly with worse declarative-episodic verbal memory in a cohort of chronic patients. Similarly, Lim et al (2006) reported that lower FA in the white matter surrounding the hippocampus was associated with worse verbal declarative memory. Taken together, our findings provide evidence for clinical and neuropsychological correlates of anterior temporal lobe white matter abnormalities in patients with schizophrenia early in the course of illness.
We also observed a significant association between higher FA in the left inferior fronto-occipital fasciculus and greater severity of hallucinations and delusions. Although this correlation was not predicted a priori, it is consistent with data from Hubl et al (2004) who reported higher white matter directionality in a lateral temporoparietal region among patients with auditory hallucinations compared to patients without auditory hallucinations and healthy control subjects. Our findings also converge with Shergill et al (2007) who recently reported that higher FA in the superior longitudinal fasciculus and anterior cingulum was associated with a propensity to experience auditory hallucinations, although FA was lower overall in patients compared to healthy volunteers. One possible interpretation of our data is that higher FA in regions involved in processing of language and/or other auditory functions could make patients more prone to experiencing positive symptoms. Alternatively, it must be acknowledged that white matter changes in this region could be the result of having positive symptoms.

Abnormalities appeared less widespread in this sample of patients with schizophrenia studied early in the course of illness compared to studies that used more chronic samples (Buchsbaum et al, 1998; Lim et al, 1999). In this regard our findings converge with Bagary et al (2003) who reported relatively circumscribed white matter abnormalities in firstepisode patients compared to healthy volunteers in the medial prefrontal cortex, insula, and uncinate fasciculus of first-episode patients compared to healthy volunteers using magnetization transfer imaging. One explanation for the finding of less widespread white matter abnormalities in our cohort of recent onset patients is that they are progressive. This possibility would be consistent with recent MRI evidence for a progressive reduction in frontal lobe white matter volume in patients with schizophrenia over an average period of 3.3 years (Ho et al, 2003). It should also be acknowledged, however, that our study may have had reduced power to detect more widespread FA anomalies among patients prone to more chronic illness. Interestingly, there was an approximate 1.5 SD difference in FA between patients and healthy volunteers across the brain white matter in the Lim et al (1999) study compared to an approximate 1.8 SD difference in FA across the white matter regions identified as significantly different between groups in the present study. Thus, while abnormalities may be less widespread in these early onset patients compared to more chronic samples, the severity of deficits between these groups appears comparable.

There were several limitations to this study that should be acknowledged. A possible limitation of voxelwise analysis is the problem of multiple comparisons and the increased risk of a Type-I error. To limit this possibility, however, we investigated FA only in the brain white matter and focused our hypotheses on the frontal and temporal lobes. In that regard it is noteworthy that we did not observe any areas of increased FA in patients despite the bias toward a Type-I error, thus strengthening the specificity of the observed findings. In addition, despite the fact that our registration algorithm has advantages over other methods (Ardekani et al, 2005), we cannot discount the possibility of image misregistration. It should also be noted that many patients in our study were receiving antipsychotic medications. 
Although the effects of antipsychotics on FA are not well understood, several prior studies reported an association between FA and antipsychotic treatment (Minami et al, 2003; Kuroki et al, 2006) as we also observed. Longitudinal DTI studies tied to standardized clinical trials will best address questions regarding possible changes in white matter integrity that are associated with antipsychotic treatment. Another study limitation is that in the absence of the DTI colormaps neuroanatomical localization of abnormalities to white matter bundles should be considered approximate.

In summary, we present evidence for temporal lobe white matter abnormalities in patients with schizophrenia, as assessed via DTI, early in the course of illness as well as data suggesting that these abnormalities have functional correlates. The observed pattern of structure-function relations and symptom correlates may be useful in constraining neurobiological models of the disorder.

\section{ACKNOWLEDGEMENTS}

This work was supported in part by grants from NARSAD (PRS) and the National Institute of Mental Health to Dr Szeszko (MH01990), Dr Bilder (MH60374), Dr Kane (MH60575), Dr Robinson (MH60004), and the NSLIJ Research Institute General Clinical Research Center (M01 RR018535).

\section{DISCLOSURE/CONFLICT OF INTEREST}

Delbert G Robinson, has served as a speaker for Janssen and received research support from Lilly, Janssen, and BristolMyers Squibb.

Todd Lencz, served as a consultant for Clinical Data Inc.

Anil K Malhotra, served as consultant, speaker and received grant support from Ortho-McNeil Janssen, BristolMyers Squibb, Pfizer, Clinical Data Inc., and AstraZeneca.

Rachel Miller, received compensation from Healthed.

John M Kane, served as a consultant and was on the advisory board for Abbott, BMS, Janssen, Lilly, Pfizer, Vanda, Wyeth, Astra-Zeneca, and Genaissance.

\section{REFERENCES}

Anderson JE, Wible CG, McCarley RW, Jakab M, Kasai K, Shenton ME (2002). An MRI study of temporal lobe abnormalities and negative symptoms in chronic schizophrenia. Schizophr Res 58: 123-134.

Antonova E, Sharma T, Morris R, Kumari V (2004). The relationship between brain structure and neurocognition in schizophrenia: a selective review. Schizophr Res 70: 117-145.

Ardekani BA, Braun M, Hutton BF, Kanno I, Iida H (1995). A fully automatic multimodality image registration algorithm. J Comput Assist Tomogr 19: 615-623.

Ardekani BA, Guckemus S, Bachman A, Hoptman MJ, Wojtaszek M, Nierenberg J (2005). Quantitative comparison of inter-subject volumetric MRI registration methods. J Neurosci Methods 142: 67-76.

Ardekani BA, Nierenberg J, Hoptman MJ, Javitt DC, Lim KO (2003). MRI study of white matter diffusion anisotropy in schizophrenia. Neuroreport 14: 2025-2029.

Bagary MS, Symms MR, Barker GJ, Mutsatsa SH, Joyce EM, Ron MA (2003). Gray and white matter brain abnormalities in first-episode schizophrenia inferred from magnetization transfer imaging. Arch Gen Psychiatry 60: 779-788.

Bartzokis G, Nuechterlein KH, Lu PH, Gitlin M, Rogers S, Mintz J (2003). Dysregulated brain development in adult men with schizophrenia: a magnetic resonance imaging study. Biol Psychiatry 53: 412-421.

Basser PJ (1995). Inferring microstructural features and the physiological state of tissues from diffusion-weighted images. NMR Biomed 8: 333-344.

Bilder RM, Bogerts B, Ashtari M, Wu H, Alvir JM, Jody D et al (1995). Anterior hippocampal volume reductions predict frontal lobe dysfunction in first episode schizophrenia. Schizophr Res 17: 47-58.

Bilder RM, Goldman RS, Robinson D, Reiter G, Bell L, Bates JA et al (2000). Neuropsychology of first-episode schizophrenia: initial characterization and clinical correlates. Am J Psychiatry 157: 549-559.

Brickman AM, Buchsbaum MS, Ivanov Z, Borod JC, Foldi NS, Hahn E et al (2006). Internal capsule size in good-outcome and poor-outcome schizophrenia. J Neuropsychiatry Clin Neurosci 18: $364-376$.

Buchsbaum MS, Friedman J, Buchsbaum BR, Chu KW, Hazlett EA, Newmark $\mathrm{R}$ et al (2006). Diffusion tensor imaging in schizophrenia. Biol Psychiatry 60: 1181-1187.

Buchsbaum MS, Tang CY, Peled S, Gudbjartsson H, Lu D, Hazlett EA et al (1998). MRI white matter diffusion anisotropy and PET metabolic rate in schizophrenia. Neuroreport 16: 425-430.

Burns J, Job D, Bastin ME, Whalley H, Macgillivray T, Johnstone EC et al (2003). Structural disconnectivity in schizophrenia: a diffusion tensor magnetic resonance imaging study. $\mathrm{Br}$ J Psychiatry 182: 439-443.

Crow TJ (1999). Twin studies of psychosis and the genetics of cerebral asymmetry. Br J Psychiatry 175: 399-401.

Davis KL, Stewart DG, Friedman JI, Buchsbaum M, Harvey PD, Hof PR et al (2003). White matter changes in schizophrenia: evidence for myelin-related dysfunction. Arch Gen Psychiatry 60: 443-456.

Forman SD, Cohen JD, Fitzgerald M, Eddy WF, Mintun MA, Noll DC (1995). Improved assessment of significant activation in functional magnetic resonance imaging (fMRI): use of a clustersize threshold. Magn Reson Med 33: 636-647.

Galaburda AM, Sanides F, Geshwind N (1978). Human brain: cytoarchitectonic left-right asymmetries in the temporal speech region. Arch Neurol 35: 812-817.

Hakak Y, Walker JR, Li C, Wong WH, Davis KL, Buxbaum JD et al (2001). Genome-wide expression analysis reveals dysregulation of myelination-related genes in chronic schizophrenia. Proc Natl Acad Sci USA 98: 4746-4751.

Hirayasu Y, McCarley R, Salisbury D, Tanaka S, Kwon J, Frumin M et al (2000). Planum temporale and heschl gyrus volume reduction in schizophrenia. Arch Gen Psychiatry 57: 692-699.

Ho BC, Andreasen NC, Nopoulos P, Arndt S, Magnotta V, Flaum M (2003). Progressive structural brain abnormalities and their relationship to clinical outcome: a longitudinal magnetic resonance imaging study early in schizophrenia. Arch Gen Psychiatry 260: 585-594.

Hof PR, Haroutunian V, Friedrich Jr VL, Byne W, Buitron C, Perl DP et al (2003). Loss and altered spatial distribution of oligodendrocytes in the superior frontal gyrus in schizophrenia. Biol Psychiatry 53: 1075-1085.

Hubl D, Koenig T, Strik W, Federspiel A, Kreis R, Boesch C et al (2004). Pathways that make voices: white matter changes in auditory hallucinations. Arch Gen Psychiatry 61: 658-668.

Jacobsen L, Giedd J, Tanrikut C, Bradey D, Donohue B, Hamburg S et al (1997). Three-dimensional cortical morphometry of the planum temporale in childhood-onset schizophrenia. Am J Psychiatry 154: 685-687. 
Kubicki M, Park H, Westin CF, Nestor PG, Mulkern RV, Maier SE et al (2005). DTI and MTR abnormalities in schizophrenia: analysis of white matter integrity. Neuroimage 26: 1109-1118.

Kubicki M, Westin CF, Maier SE, Frumin M, Nestor PG, Salisbury DF et al (2002). Uncinate fasciculus findings in schizophrenia: a magnetic resonance diffusion tensor imaging study. $A m \mathrm{~J}$ Psychiatry 159: 813-820.

Kubicki M, Westin CF, Nestor PG, Wible CG, Frumin M, Maier SE et al (2003). Cingulate fasciculus integrity disruption in schizophrenia: a magnetic resonance diffusion tensor imaging study. Biol Psychiatry 54: 1171-1180.

Kumra S, Ashtari M, Cervellione KL, Henderson I, Kester H, Roofeh D et al (2005). White matter abnormalities in early-onset schizophrenia: a voxel-based diffusion tensor imaging study. J Am Acad Child Adolesc Psychiatry 44: 934-941.

Kuroki N, Kubicki M, Nestor PG, Salisbury DF, Park HJ, Levitt JJ et al (2006). Fornix integrity and hippocampal volume in male schizophrenic patients. Biol Psychiatry 60: 22-31.

Kwon J, McCarley R, Hirayasu Y, Anderson J, Fischer I, Kikinis R et al (1999). Left planum temporale volume reduction in schizophrenia. Arch Gen Psychiatry 56: 142-148.

Lencz T, Smith CW, McLaughlin D, Auther A, Nakayama E, Hovey L et al (2006). Generalized and specific neurocognitive deficits in prodromal schizophrenia. Biol Psychiatry 59: 863-871.

Lim KO, Ardekani BA, Nierenberg J, Butler PD, Javitt DC, Hoptman MJ (2006). Voxelwise correlational analyses of white matter integrity in multiple cognitive domains in schizophrenia. Am J Psychiatry 163: 2008-2010.

Lim KO, Hedehus M, Moseley M, de Crespigny A, Sullivan EV, Pfefferbaum A (1999). Compromised white matter tract integrity in schizophrenia inferred from diffusion tensor imaging. Arch Gen Psychiatry 56: 367-374.

MEDx (2000) Sensor Systems Inc: Sterling, VA.

Meng X, Rosenthal R, Rubin DB (1992). Comparing correlated correlation coefficients. Psychol. Bull 111: 172-175.

Minami T, Nobuhara K, Okugawa G, Takase K, Yoshida T, Sawada $S$ et al (2003). Diffusion tensor magnetic resonance imaging of disruption of regional white matter in schizophrenia. Neuropsychobiology 47: 141-145.

Mitelman SA, Torosjan Y, Newmark RE, Schneiderman JS, Chu $\mathrm{KW}$, Brickman AM et al (2007). Internal capsule, corpus callosum and long associative fibers in good and poor outcome schizophrenia: a diffusion tensor imaging survey. Schizophr Res 92: 211-224

Mori S, Wakana S, Nagae-Poetscher LM, van Zijl PCM (2005). MRI Atlas of Human White Matter. Elsevier Science: London.

Nestor PG, Kubicki M, Gurrera RJ, Niznikiewicz M, Frumin M, McCarley RW et al (2004). Neuropsychological correlates of diffusion tensor imaging in schizophrenia. Neuropsychology 18: 629-637.

Park HJ, Westin CF, Kubicki M, Maier SE, Niznikiewicz M, Baer A et al (2004). White matter hemisphere asymmetries in healthy subjects and in schizophrenia: a diffusion tensor MRI study. Neuroimage 23: 213-223.
Rhinewine JP, Lencz T, Thaden EP, Cervellione KL, Burdick KE, Henderson I et al (2005). Neurocognitive profile in adolescents with early-onset schizophrenia: clinical correlates. Biol Psychiatry 58: 705-712.

Robinson DG, Woerner MG, Napolitano B, Patel RC, Sevy SM, Gunduz-Bruce $\mathrm{H}$ et al (2006). Randomized comparison of olanzapine versus risperidone for the treatment of first-episode schizophrenia: 4-month outcomes. Am J Psychiatry 163: 2096-2102.

Ruchsow M, Trippel N, Groen G, Spitzer M, Kiefer M (2003). Semantic and syntactic processes during sentence comprehension in patients with schizophrenia: evidence from event-related potentials. Schizophr Res 64: 147-156.

Shergill SS, Kanaan RA, Chitnis XA, O'Daly O, Jones DK, Frangou $S$ et al (2007). A diffusion tensor imaging study of fasciculi in schizophrenia. Am J Psychiatry 164: 467-473.

Sigmundsson T, Suckling J, Maier M, Williams S, Bullmore E, Greenwood $\mathrm{K}$ et al (2001). Structural abnormalities in frontal, temporal, and limbic regions and interconnecting white matter tracts in schizophrenic patients with prominent negative symptoms. Am J Psychiatry 158: 234-243.

Szeszko PR, Ardekani BA, Ashtari M, Kumra S, Robinson DG, Sevy S et al (2005a). White matter abnormalities in first-episode schizophrenia or schizoaffective disorder: a diffusion tensor imaging study. Am J Psychiatry 162: 602-605.

Szeszko PR, Babak AA, Ashtari M, Malhotra AK, Robinson DG, Bilder RM et al (2005b). White matter abnormalities in obsessive-compulsive disorder: a diffusion tensor imaging study. Arch Gen Psychiatry 62: 782-790.

Szeszko PR, Bilder RM, Dunlop J, Walder DJ, Lieberman JA (1999). Longitudinal assessment of methylphenidate effects on oral word production in acute and stabilized patients with firstepisode schizophrenia. Biol Psychiatry 45: 680-686.

Szeszko PR, Goldberg E, Gunduz-Bruce H, Ashtari M, Robinson D, Malhotra AK et al (2003). Smaller anterior hippocampal formation volume in antipsychotic-naive patients with firstepisode schizophrenia. Am J Psychiatry 160: 2190-2197.

Szeszko PR, Strous RD, Goldman RS, Ashtari M, Knuth KH, Lieberman JA et al (2002). Neuropsychological correlates of hippocampal volumes in patients experiencing a first episode of schizophrenia. Am J Psychiatry 159: 217-226.

Tkachev D, Mimmack ML, Ryan MM, Wayland M, Freeman T, Jones PB et al (2003). Oligodendrocyte dysfunction in schizophrenia and bipolar disorder. Lancet 362: 798-805.

Uranova N, Orlovskaya D, Vikhreva O, Zimina I, Kolomeets N, Vostrikov V et al (2001). Electron microscopy of oligodendroglia in severe mental illness. Brain Res Bull 55: 597-610.

Wang F, Sun Z, Cui L, Du X, Wang X, Zhang H et al (2004). Anterior cingulum abnormalities in male patients with schizophrenia determined through diffusion tensor imaging. $\mathrm{Am} \mathrm{J}$ Psychiatry 161: 573-575.

Wright IC, Rabe-Hesketh S, Woodruff PW, David AS, Murray RM, Bullmore ET (2000). Meta-analysis of regional brain volumes in schizophrenia. Am J Psychiatry 157: 16-25. 